\title{
QUELQUES PRÉCISIONS SUR LES CARACTÈRES SPÉCIFIQUES \\ DU STRONGLE GÉANT DU CHIEN
}

\author{
Par le Dr WITOLD STEFANSKI \\ Professeur de Zoologie et Parasitologie à la Faculté vétérinaire de Varsovie
}

Yorke et Maplestone, dans leur excellent ouvrage sur les nématodes parasites des vertébrés (1926), parlent de l'existence des six papilles buccales chez Dioctophyme Collet-Meygret, 1802, en opposition avec le genre Eustrongylides Jaegerskiöld, 1909, lequel est muni de 12 papilles buccales (ou 18), disposées en deux cercles. Dans la diagnose du genre, nous lisons également: " ...mouth simple without lips, but surrounded with a circle of six papillæ ".

Cette notion erronée sur l'existence des six papilles buccales se répète chez tous les auteurs (sauf chez Baylis et Daubney, 1926), malgré l'autorité de Leuckart, lequel dans sa diagnose du genre s'exprime ainsi : “ Der Körper ist am Hinterende abgestumft, vorn dagegen, besonders bei dem Männchen, mehr zugespitz und im umkreis der Undeutlich sechseckigen Mundœffnung mit sechs warzenförmigen Papillen von ansehnlicher Grösse besetzt, neben denen noch mehrere andere kleinere gefunden werden. "

Nous n'avons jusqu'à présent qu'un seul travail dans lequel les caractères systématiques et l'anatomie de notre ver soient traités d'une manière plus détaillée, c'est eelui de Leuckart (1876). Et on peut même s'étonner que l'auteur en question n'ayant à sa disposition qu'un matériel très restreint et mal fixé en ait su tirer un si bon parti.

D'autre part, Balbiani (1870) a consacré son attention à la question -du développement sans réussir malheureusement à l'élucider.

Nombre de questions demandent cependant à être reprises et travaillées avec les méthodes modernes, pour ne parler que de la structure. de l'œsophage sur la signification de laquelle Leuckart s'est visiblement mépris.

D'autre part, il est d'une importance capitale, pour la connaissance du cycle évolutif, d'être fixé sur la spécificité des strongles, provenant de différents hôtes, appartenant, comme nous savons, à des groupes systématiques très éloignés: phoque, loutre, putois, loup, chien, bœuf, cheval, pore et homme.

La facilité relative avec laquelle on peut se procurer à Varsovie Annales de Parasitologie, t. VI, ${ }^{\circ} 1 .-1^{\text {er }}$ janvier 1928 , p. 93-100. 
des strongles géants du chien m'a décidé enfin d'entreprendre l'étude plus approfondie de ce ver en commençant par les caractères externes.

Mes observations sont basées sur l'examen de 11 individus dont 6 mâles et 5 femelles (1). Sur ce nombre, 4 mâles et 4 femelles provenaient du rein et deux mâles et une femelle de la cavité abdominale ou plus exactement de la région située entre les lobes du foie.

Notons dès à présent, pour éviter la répétition dans la description, que l'identité spécifique des individus provenant de ces deux endroits ne peut pas être mise en doute.

Femelle. - Longueur du corps : $673 \mathrm{~mm}$. (260-760 mm.). Diamètre du corps : $7 \mathrm{~mm}$., 5 (5-8 mm.), soit $\alpha=89,7$. Longueur de l'œsophage : $52 \mathrm{~mm}$. (44-60 mm.), soit $\beta=13$. Distance de la vulve : $42 \mathrm{~mm}$. (14-65 mm.), soit $\mathrm{V}=60 / 0(5,40 / 0-8,50 / 0)$. La couleur rouge sang varie probablement selon l'âge de l'animal, les individus plus jeunes ayant une teinte plus claire et les individus plus âgés ayant une couleur plus foncée. La couleur devient également plus foncée, mais sale en même temps, au moment de Ia mort du ver.

La forme générale du corps est celle d'un grand nématode typique, peu atténuée dans la partie antérieure et dont le diamètre reste presque le même sur toute la longueur, l'extrémité postérieure étant obtuse.

La cuticule est transversalement striée mais d'une manière assez superficielle. La largeur des stries est de $0 \mathrm{~mm}$., 045-0 mm., 06.

Sur les lignes latérales la cuticule porte deux rangées régulières de papilles dont l'existence fut relevée pour la première fois par Leuckart. Ce sont de petites papilles visibles déjà à l'œil nu, surtout sur le corps non mouillé par le liquide, mais qui dépassent à peine la surface de la cuticule. Leur hauteur, depuis leur base, est de $0 \mathrm{~mm} ., 075$, tandis que l'épaisseur de la cuticule est de $0 \mathrm{~mm}$., 06 et leur diamètre, à la base, mesure $0 \mathrm{~mm}$., 06 . Sous le microscope et lorsque l'objet est bien éclairci, on voit pénétrer la pulpe à l'intérieur de la papille.

Le nombre de papilles varie suivant la longueur de l'individu depuis 150-170. La rangée des papilles latérales commence à une petite distance de la bouche, s'étendant jusqu'au voisinage de l'anus. Dans la région céphalique, les papilles sont distribuées d'une manière très serrée, de façon à ce que la distance qui les sépare ne dépasse pas $1 / 3$ de millimètre. Plus on avance vers le milieu, plus la

(1) Ce matériel m'a été gracieusement fourni par M. le prof. C. Janicki dans le laboratoire duquel on travaille également sur certains problèmes concernant ce nématode, ainsi que par $\mathrm{M}$. le prof. W. Roszkowski. Je tiens à leur exprimer ici à tous deux mes vifs remerciements. 
distance entre elles devient grande, passant successivement à $0 \mathrm{~mm}$., 5, 2, 3, 4, 5 et $6 \mathrm{~mm}$. Suivant les individus, on les voit plus ou moins distinctement, mais d'habitude au niveau de la $112^{\circ}$ ou de la $115^{\circ}$ papille (sur la distance de $30 \mathrm{~mm}$.) celles-ci deviennent peu distinctes. A cet endroit, les papilles s'espacent de plus en plus et enfin cette distance s'élève jusqu'à $55 \mathrm{~mm}$. entre la $119^{\circ}$ et la $120^{\circ}$ papille. C'est le milieu du corps. Au delà du milieu du corps, les papilles se resserrent de nouveau et leur distance l'une de l'autre diminue progressivement.

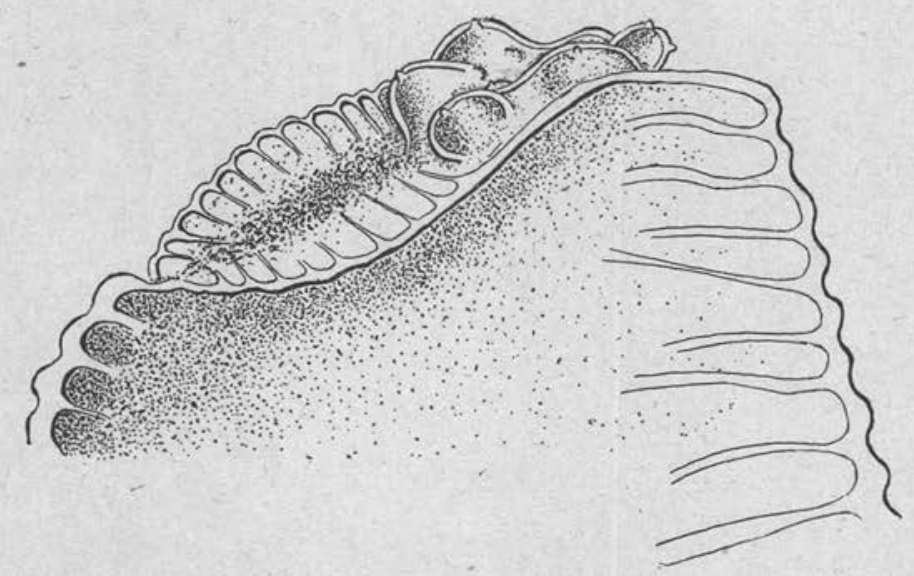

FıG. 1. - Dioctophyme renale (Goeze, 1782). Région céphalique, vue du côté gauche, montrant la disposition des nodules et des papilles, $\times 42$.

Les deux lignes latérales ne sont pas toujours symétriques à ce point de vue et quelquefois, sur le côté droit par exemple, le nombre de papilles est plus grand et la distance qui les sépare, au milieu du corps, est plus petite que sur le côté gauche (1).

Dans la région céphalique (fig. 1 et 2 ), on trouve du côté ventral une dépression longitudinale. Les lèvres de cette dernière s'élèvent de deux côtés, couvrant en partie les nodules latéraux et ventraux ; ces derniers sont en outre situés plus bas que les autres et se trouvent dans la dépression mème. La région nodulaire est ainsi fortement rétrécie par rapport au diamètre de la région voisine.

En ce qui concerne les nodules mêmes, ils sont disposés de

(1) La disposition des papilles latérales est du reste sujette à des variations assez étendues; ainsi Leuckart $(l$. c.) trouve chez son plus grand exemplaire, au milieu du corps, que la plus grande distance entre les papilles ne dépasse pas $4 \mathrm{~mm}$. à $5 \mathrm{~mm} ., 5$. 
manière à ce que deux d'entre eux correspondent aux lignes latérales, tandis que les quatre autres se disposent symétriquement de deux côtés des lignes médianes, ventrale et dorsale. Leur diamètre varie suivant les dimensions de l'individu, entre $0 \mathrm{~mm} ., 16$ et $0 \mathrm{~mm} ., 25$ et leur hauteur entre $0 \mathrm{~mm} ., 1$ et $0 \mathrm{~mm} ., 15$. Chaque nodule est pourvu à son sommet d'une véritable papille où on voit nettement converger les fibres nerveuses. Le diamètre de la papille qui, vue d'en haut, se projette en forme de cercle, ne dépasse pas $0 \mathrm{~mm}$., 03 et sa hauteur atteint $0 \mathrm{~mm}$, 015. Le nodule dorsal gauche porte en opposition aux deux autres, deux de ces papilles, caractère qui me semble constant, bien qu'il me soit impossible de le constater chez tous les individus.

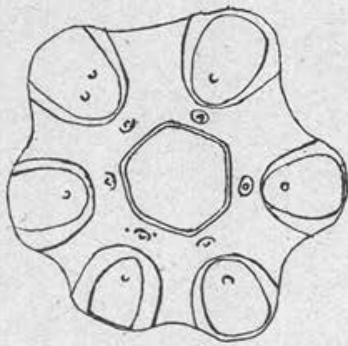

FIG: 2. - Diagramme montrant la disposition des nodules et des papilles chez Dioctophyme renale (Goeze, 1782).

A la base des nodules, du côté de l'orifice buccal, on voit s'élever encore six autres nodules beaucoup plus petits, du diamètre de $0 \mathrm{~mm}$., 07, et portant une minuscule papille. Mentionnons encore que, du côté externe des nodules latéraux, se trouve une paire de petites papilles, situées cependant en dehors de la région céphalique et qui pourraient être plutôt assimilées aux papilles cervicales. Leuckart parle de la disposition de toutes ces papilles en termes très clairs : "Diese sechs Papillen (Leuckart parle du cercle externe) sind aber keineswegs die einzigen, die am Kopfende unseres Wurmes vorkommen. Es geselle sich zu ihnen noch andere, die freilich nur bei genaurerer Untersuchung gesehen worden. So steht zunächst an dem oralen Rande der sechs grossen Hervorragungen, je noch ein kleiner Höcker von etwa $0,08 \mathrm{~mm}$., der sich bei mikroskopischer Untersuchung gleichfalls als eine Papille aufweist. Auch an den benachbarten Seitenrändern sind ein Paar derartige Höckerchen entwickelt, nur wie es scheint nicht so constont an allen Hervorragungen. Ich sehe sie vornähmlich an den lateralen Papillen. " 
Malheureusement le cercle des papilles internes ne figure pas sur le dessin de Leuckart (fig. 205) et c'est ainsi qu'il faut expliquer leur omission dans la diagnose des auteurs.

Parmi les exemplaires examinés, la région céphalique d'un mâle (1 sur 6), provenant de la cavité abdominale, montrait la particularité suivante : la partie supérieure des nodules était kératinisée, les papilles faisaient défaut, le cercle papillaire interne étant à ce point de vue normal. Je ne peux pas attacher à ce phénomène une importance spécifique, le considérant plutôt comme un cas anormal, les autres caractères restant typiques.

L'orifice anal se trouve à l'extrémité postérieure du corps, de sorte que la queue fait défaut. Sa forme est semi-lunaire et son grand axe $(0 \mathrm{~mm} ., 96)$ est placé entre les deux lignes latérales. La lèvre dorsale de l'anus surmontant la lèvre ventrale est un peu échancrée du côté droit à cause du développement à cet endroit du bourrelet latéral droit.

Ainsi que Leuckart le fait remarquer, les rangées de papilles latérales se prolongent dans la région anale, se disposant en deux ares autour de l'orifice. Je n'ai trouvé pour ma part que deux de ces papilles de chaque côté de l'orifice et dirigées vers la face ventrale.

MaLe. - Longueur du corps : $373 \mathrm{~mm}$. (295-450); diamètre du corps : $3 \mathrm{~mm}$., 7 (3-4). La forme générale du corps ainsi que la couleur sont les mêmes que chez la femelle, le mâle étant évidemment plus svelte $(\alpha=100)$. Dans la région céphalique, on trouve le même nombre et la même disposition des papilles buccales, ces dernières étant plus petites. Ainsi le diamètre des nodules externes est de $0 \mathrm{~mm}$., 15 et celui des papilles de $0 \mathrm{~mm}$., 022 . De même, le diamètre des nodules internes est de $0 \mathrm{~mm}$., 045 et celui des papilles de 0 mm., 009.

Par contre, la bourse copulatrice (fig. 3 et 4 ) est construite d'une manière très particulière et avait déjà attiré l'attention des premiers auteurs qui ont étudié ce nématode. La forme générale de cette bourse est celle d'une cloche ovale dont le grand axe (3 mm., 75) s'allonge latéralement. A sa naissance, l'extrémité caudale présente un rétrécissement de $2 \mathrm{~mm}$., 1, ce qui ne constitue point un étranglement brusque et ne peut par conséquent servir de pivot pour la bourse copulatrice. Celle-ci est plus haute du côté dorsal et ne présente qu'une légère échancrure médiane. La hauteur de la bourse atteint à cet endroit $2 \mathrm{~mm}$., 1. Du côté ventral, la bourse est beaucoup plus basse et sa hauteur maxima est de $0 \mathrm{~mm}$., 75. Je dis maxima, car la plus grande partie de la lèvre inférieure est constituée par des parois plus minces que sa base et 
pouvant se contracter, en découvrant de cette façon le fond de la bourse. La distance entre les deux lèvres atteint $2 \mathrm{~mm}$., 8 . Le fond de la bourse n'est pas plat, ainsi que le fait remarquer Leuckart dans la diagnose de l'espèce (p. 355) : " Der grund der Bursa wird von einer Y-förmigen Erhebung durchzogen, deren unpaarer Schenkel nach der Mitte des Vorderrandes gekehrt ist. We die

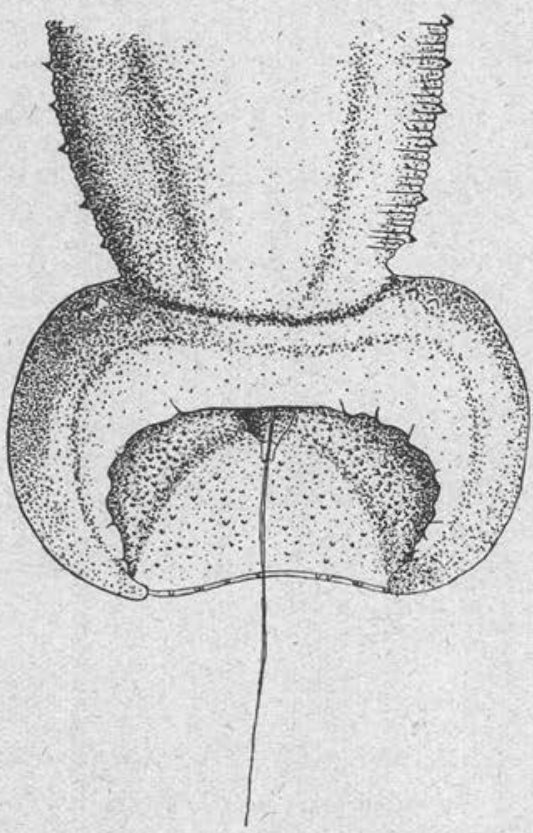

Fig. 3. - Dioctophyme renale (Goeze, 1782). Bourse copulatrice vue du côté ventral, $\times 21$.

beiden hinteren Schenkel zusammenkommen, findet sich auf der Spitze eines kleinen Höckers die Kloaköffnung... "

En effet, le fond de la bourse, du côté de sa paroi dorsale, s'élève en un bourrelet large de $1 \mathrm{~mm}$., 5 et s'étendant sur une longueur de $0 \mathrm{~mm}$., 5. Des deux angles supérieurs de ce bourrelet partent en divergeant deux ailes, larges de 0 mm., 4, aboutissant jusqu'aux bords supérieurs de la bourse. Au milieu du bourrelet impair s'élève un cône cloacal dont la hauteur varie entre $0 \mathrm{~mm}$., 3 et $0 \mathrm{~mm}$., 37 et dont le diamètre à la base est presque double. La partie supérieure de ce cône est séparée par un rétrécissement superficiel, circulaire. 
Voici comment Leuckart s'exprime à propos des papilles bursales (p. 376) : " Ebense kennen wir bereits die Existenz zahlreicher Fühlpapillen, die in unregelmässigen Entfernungen von 0,04-0,1 mm. neben einander den Rand der Bursa Kranzförmig umgeben, aber nur klein sind, so dass sie ohne starke Vergrösserung kaum bemerkt werden können. "

Or, en cherchant à me rendre compte de la distribution de ces papilles et en mettant à cet effet la partie caudale du ver dans

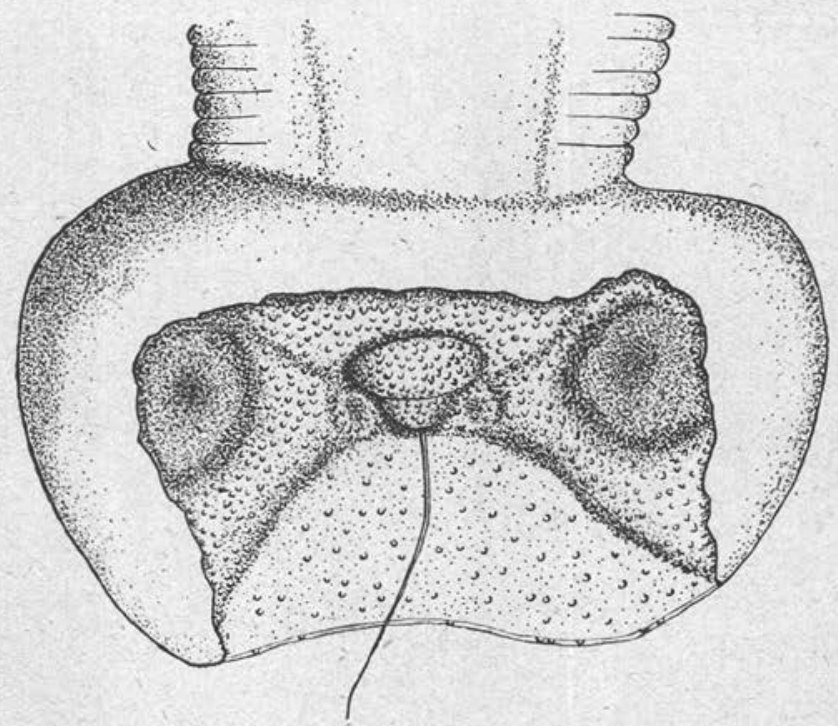

Fig. 4. - Dioctophyme renale (Goeze, 1782). Bourse copulatrice vue du côté ventral; lèvre ventrale enlevée pour montrer le fond de la bourse avec les bourrelets couverts de papilles et le cône cloacal, $\times 30$.

l'acide lactique, je fus bientôt étonné de trouver tout le cône cloacal couvert de minuscules papilles et, à mesure que l'éclaircissement de la préparation s'avançait, d'autres papilles apparurent. Voici dans quel ordre elles sont distribuées.

La rangée papillaire latérale s'étend sur la bourse mais s'interrompt déjà à une courte distance, n'étant plus représentée que par deux paires de papilles. Viennent ensuite des papilles de bordure, distribuées d'une manière fort irrégulière sur le bord du calice bursal. Il y en a environ 25 , ce nombre pouvant varier suivant les individus. La distance qui les sépare varie entre $0 \mathrm{~mm}$., 04 à $0 \mathrm{~mm} ., 46$.

Le fond du calice bursal est couvert de très nombreuses papilles 
de $0 \mathrm{~mm} ., 015$ de hauteur chacune. Elles sont situées en grand nombre sur les bourrelets mentionnés et ressortent d'autant mieux sur le fond que le reste de la bourse n'en présente qu'en quantité beaucoup moindre. De même, le cône cloacal est entouré de 12 rangées papillaires.

Il est étonnant que Leuckart n'ait pas vu les papilles couvrant le fond de la bourse et cela d'autant plus qu'il décrit les bourrelets d'une manière très exacte. Son unique exemplaire mâle provenait du vison. S'agirait-il d'une autre espèce ?

La bourse n'est pas susceptible de déplacer son axe par rapport à l'axe principal de l'animal, par contre elle peut fonctionner efficacement comme une ventouse, en contractant plus ou moins sa lèvre inférieure. Les ailes (côtes) s'élevant du fond de la bourse contribuent à renforcer l'adhérence de cette ventouse au corps de la femelle.

\section{RÉSUMÉ}

1. L'orifice buccal dans le genre Dioctophyme Collet-Meygret, 1802 est entouré de deux rangées de nodules, six dans chaque rangée, portant le même nombre de papilles à leur sommet.

2. Les individus, provenant de la cavité abdominale et ceux qui proviennent du rein du chien n'accusent aucune différence spécifique,

3. Le fond de la bourse copulatrice est couvert de très nombreuses papilles, distinctes surtout sur les bourrelets.

4. L'existence de ce dernier caractère omis dans la description du mâle étudié par Leuckart, et provenant du vison, permet d'émettre des doutes sur l'identité spécifique des parasites du chien et de ceux du vison.

\section{BiBLIOGRAPHIE}

BaLbiani (M.). - Recherches sur le développement et la propagation du strongle géant. Recueil méd. vétér., XLVII et Journ. anat. et physiol., VII, 1870.

Baylis et Daubnex. - A Synopsis of the families and genera of Nematoda. London, 1926.

Leuckart (R.). - Die menschlichen Parasiten und die von ihnen herrührenden Krankheiten, II, Leipzig und Heidelberg, 1876.

Yorke (W.) et Maplestone (P.-A.). - The Nematode parasites of Vertebrates, London, 1926.

Laboraloire de Zoologie el de Parasitologie de la Faculté vétérinaire de Varsovie. 